\title{
Wavefront Controls for a Large Submillimeter-Wave Observatory
}

\author{
David Redding ${ }^{* a}$, John Z. Lou ${ }^{\mathrm{a}}$, Andy Kissil ${ }^{\mathrm{a}}$, Matt Bradford ${ }^{\mathrm{a}}$, Steve Padin ${ }^{\mathrm{b}}$ and David Woody ${ }^{\mathrm{b}}$ \\ ${ }^{a}$ Jet Propulsion Laboratory, California Institute of Technology, Pasadena CA 91109; \\ ${ }^{\mathrm{b}}$ California Institute of Technology, Pasadena CA 91106
}

\begin{abstract}
The 25-m aperture Cornell Caltech Atacama Telescope (CCAT) will have a primary mirror that is divided into 162 individual segments, each of which is equipped with 3 positioning actuators. This paper presents a mathematical description of the telescope, its actuators and sensors, and uses it to derive control laws for figure maintenance. A Kalman Filter-based Optical State Estimator is used to continuously estimate the aberrations of the telescope; these are used in a state-feedback controller to maintain image quality. This approach provides the means to correct for the optical effects of errors that occur in un-actuated degrees of freedom, such as lateral translations of the segments. The control laws are exercised in Monte Carlo and simulation analysis, to bound the closed-loop performance of the telescope and to conduct control design trades.
\end{abstract}

Keywords: Segmented mirror, wavefront control, edge sensors, Kalman filter.

\section{INTRODUCTION}

The 25-m aperture Cornell Caltech Atacama Telescope (CCAT) will be sited at an altitude of $5600 \mathrm{~m}$ in the Atacama Desert, operating at wavelengths between $200 \mathrm{um}$ and $2 \mathrm{~mm}$ [1]. Its primary mirror will be assembled from 162 "keystone" segments, each of which is equipped with 3 rigid-body actuators. These will be used to move the segments, so as to counter deformations from temperature changes and the varying gravity effects that come with pointing changes. The baseline design will utilize a steel structure, with active figure controls to establish and maintain good optical performance. The telescope figure will be initialized using submillimeter-wave shearing interferometry [2] to determine the best positioning of the segments.

This paper examines the problem of active controls to maintain good optical figure once it has been initially established. It describes a Wavefront Compensation controller that uses innovative, inexpensive optical edge sensors to measure segment-to-segment displacements and determine actuator commands that maintain good optical quality. The controller uses a Kalman filter to estimate the full optical state of the telescope, including 6 degrees of freedom (DOF) of change for each segment, and to predict the Wavefront Error (WFE) that results from these changes. Actuator controls are computed to minimize this WFE at each time step, using only the 3 DOF provided by the actuators. This approach is able to partly compensate the WFE due to motions in the uncontrolled DOFs, resulting in better performance than approaches that simply seek to null the sensor signals.

The estimator and controller are based on a detailed mathematical model of the telescope, currently based on structural and optical computer models, but which will ultimately be determined by direct calibration. These models are summarized in Section 3, following a summary of nomenclature in Section 2. The estimator and controller are derived in Sections 4-6, and example results are presented in Section 7.

\section{NOMENCLATURE}

$x_{R}$, rigid-body state of each optic or optical assembly, $\left[x_{R}\right]=\left(6^{*}\right.$ nopt, 1$)$

$x_{D}$, segment deformation state, $\left[x_{D}\right]=($ ndef*nseg, 1$)$

$u_{R}$, segment rigid body control command, $\left[u_{R}\right]=(6 *$ nseg, 1$)$

$u_{S M}$, SM rigid body control command, $\left[u_{S M}\right]=(5,1)$

$u_{c}$, pointing control command, $\left[u_{c}\right]=(2,1)$

$\delta T$, temperature change, $[\delta T]=\left(\mathrm{nT}^{*} \mathrm{nseg}, 1\right)$

Ground-based and Airborne Telescopes III, edited by Larry M. Stepp, Roberto Gilmozzi, Helen J. Hall Proc. of SPIE Vol. 7733, 773329 - (C) 2010 SPIE - CCC code: 0277-786X/10/\$18 - doi: 10.1117/12.858097 
$x$, process noise, $[d T]=(x, 1)$

$l$, optical edge sensor measurements, $[l]=(2 * \mathrm{nl}, 1)$

$\delta l$, optical edge measurement noise, $[\delta l]=(2 * n l, 1)$

$w$, wavefront, $[w]=($ nray, 1$)$

$w_{A t m}$, wavefront error due to atmosphere

$\delta w$, wavefront measurement noise, $[\delta w]=($ nray, 1$)$

$O P D$, Optical Path Difference matrix

$c$, ray centroid, $[c]=(2,1)$

$\delta c$, pointing measurement noise, $[\delta c]=(2,1)$

$K$, estimator gain

$c_{w u}$, control weighting coefficient

$i$, time index

Capitalized variable signifies the covariance of that variable

Bar over a variable signifies a predicted value (prior to incorporation of current measurement data)

Hat over a variable signifies current estimate

Squiggle over a variable signifies current estimate error

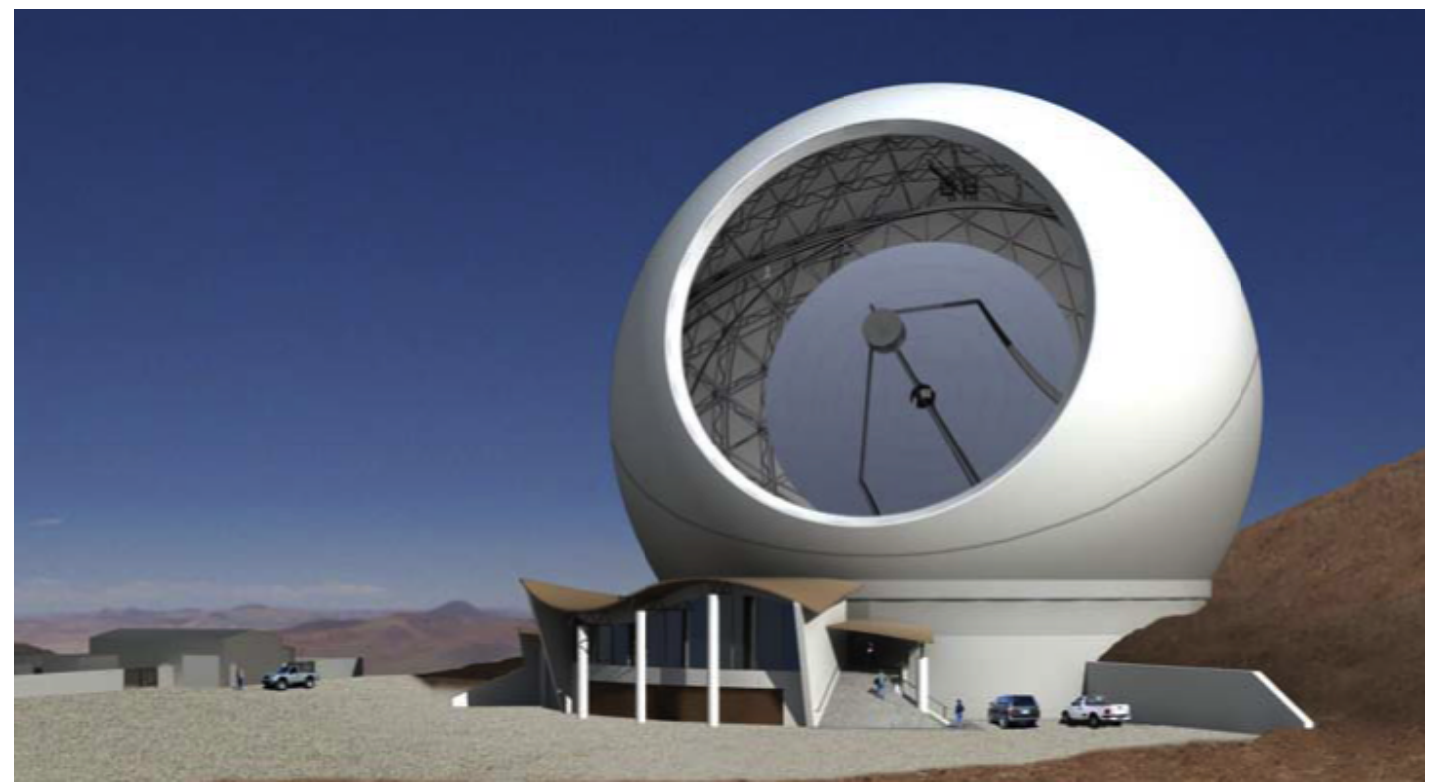

Figure 1. CCAT overview.

\section{CCAT MODEL}

A detailed integrated optics/structures/thermal computer model of CCAT is being built, to aid in design studies of the CCAT telescope [3]. The optics part of the CCAT integrated model has been built using the JPL MACOS code [4]. This model includes each optic, i.e., 162 primary mirror (PM) segments, the secondary mirror (SM), back-end optics and detector, as a separate body capable of displacement in 3 rotational and 3 translational DOFs. Perturbations from their ideal designed positions and orientations are captured in the "rigid-body optical state," $x_{R i}$, a vector of dimension $948 \times 1$, evaluated at the $i^{\text {th }}$ time interval. The state is defined in coordinates local to each optic, centered at the pole of the optic, oriented along the local radial, tangential and normal directions, relative to the commanded global telescope pointing.

The optics have thermal deformations and figure errors due to fabrication errors, which effects are included in the "deformation optical state," $x_{D i}$. Thermal modes are computed using the structures part of the CCAT integrated model, using NASTRAN. The thermal modes are used to compute optical surface deformations at node points on a grid defined in local coordinates for each optic. The total optical state vector combines these elements into a single vector. 
The full state at the $i^{\text {th }}$ time interval combines the rigid and deformation states of all of the optics into a single vector:

$$
x_{i}=\left[\begin{array}{l}
x_{R i} \\
x_{D i}
\end{array}\right]
$$

Each segment is equipped with 3 actuators, which control its tip, tilt and piston in the same local coordinate frames. Actuator-driven segment perturbations are denoted $u$. The SM is equipped with 6 actuators; its perturbations are $u_{S M}$.

The full dynamics of the CCAT structure are not included in the control derivation model, though ultimately they will be included in a more detailed system performance simulation: the "truth model." For control design purposes it is sufficient to envelope the behavior of the CCAT structure, using a process noise term $\xi$ to introduce random state changes; and using coupled disturbance modes $T$, including thermal modes of the segments, thermal modes of the telescope structures, the effects of gravity as the telescope pointing changes, global pointing errors, and other global effects. This results in a state transition equation that does not include any dynamics:

$$
x_{i}=x_{i-1}+\frac{\partial x}{\partial u} u_{i}+\frac{\partial x}{\partial u_{S M}} u_{S M i}+\frac{\partial x}{\partial T} T_{i}+\xi_{i}
$$

That is, the state at the $i+1^{\text {th }}$ time interval is equal to the state at the $i^{\text {th }}$ time interval, plus changes induced by actuator motions, changes due to disturbance modes, and changes due to the process noise $\xi$. The coupling of the actuations to the state are through the $\partial x / \partial u$, which are simple mapping matrices. The mode sensitivities $\partial x / \partial T$ are generated using the CCAT FEA model. Global modes $T$ include segment temperature soaks, substructure gradients, substructure soaks, gravity sag at different elevation angles, and pointing errors. The $T$ are used to provide initial conditions for the state; they are also varied with time step $i$ to provide bounding disturbances for the CCAT controller worst-case analysis.

Changes in the state $x$ have optical effects: wavefront $w$ degrades; the telescope pointing $c$ wanders. The CCAT MACOS model computes these optical effects by perturbing the position and shape of each optic, and then tracing rays through the optical system, from a star at infinity, past each optic to the detector. The wavefront $w$ is the vectorized form of the Optical Path Difference (OPD) matrix: a map of the wavefront (WF) across the pupil, evaluated at an exit pupil conjugate to the PM, which defines the entrance pupil - see Figure 2. The pointing error centroid $c$ is computed as the centroid of all of the rays at the detector. The MACOS model includes atmospheric seeing effects $w_{A t m}$ and $c_{A t m}$ as well.
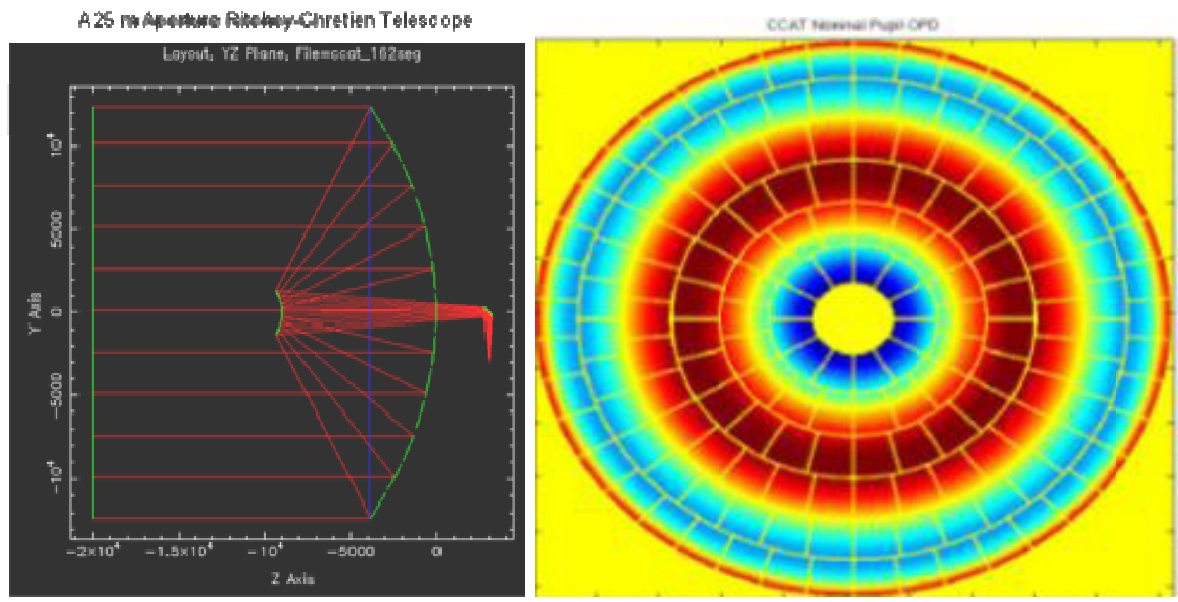

Figure 2. CCAT ray-trace (left) and nominal OPD showing PM segmentation; wavefront $w$ is vectorized OPD.

For the small motions typical of a controlled telescope, the MACOS model can be linearized, and the effect of the state on the optics can be expressed in matrix form, as:

$$
w_{i}=\frac{\partial w}{\partial x} x_{i}+w_{A t m}+w_{0}
$$




$$
c_{i}=\frac{\partial c}{\partial x} x_{i}+c_{A t m}+c_{0}
$$

That is, the WF $w$ is equal to the nominal WF $w_{0}$ plus the optical effects of the perturbations $x$, plus the effects of atmospheric turbulence $w_{A t m}$ (small in the submillimeter); similarly for the pointing $c$. Here MACOS is used to generate the optical sensitivities $\partial w / \partial x$ and $\partial c / \partial x$, following the theory of [5]. This provides linear optical models that predict the WFE and pointing error using matrix operations rather than a full ray-trace; such models are highly accurate for the small state excursions typical of a controlled telescope. The optical sensitivities are also important in the derivations of the controller - see below. The RMS Wavefront Error (WFE) is computed as the RMS of $w_{i}$.

Changes in the state $x$ also impact the outputs of the measurements that are used for control. In this paper we consider 3 types of measurement. The first is a direct measurement of the WFE, which can be obtained by WF sensing, or using the shearing interferometry technique discussed in [2]. The WF measurement equation takes the form (assuming fully unwrapped phase):

$$
w_{m i}=w_{i}+\delta w_{i}=\frac{\partial w}{\partial x} x_{i}+w_{A t m}+w_{0}+\delta w
$$

The second type of measurement is made using optical edge sensors. The CCAT edge sensors have 2 parts: a collimated light source located on one segment; and a CCD camera located on the adjacent segment - see Figure 3. Translational or rotational motions of one segment relative to the other cause lateral displacement of the light beam, which is measured by the CCD in 2 axes: in the "z" or local surface normal direction; and "y," parallel to the mirror surface, as described in [6] and [7]. Optical edge sensors are placed as shown in Figure 3, with approximately 2 sensors per segment-to-segment edge, and a total of 624 sensors, each sensing in 2 axes for a total of 1248 measurements. Design parameters governing edge sensor performance include the mounting position below the surface and the radial position of each sensor.

The edge sensor measurement equation takes the form:

$$
l_{i}=\frac{\partial l}{\partial x} x_{i}+l_{0}+\delta l_{i}
$$

Here the $\partial l / \partial x$ sensitivities capture the kinematics of the sensors. The $l_{0}$ vector represents the static offset of the edge sensors, which can be calibrated. Optical edge sensor measurements will be made at a frequency of $0.1 \mathrm{to} 1 \mathrm{~Hz}$.

The third type of measurement is the telescope pointing measurement, made using an off-axis guide star and a separate pointing camera. For this analysis, we assume that the pointing camera provides a measurement to be directly compared to an image at the center of the CCAT field. Unlike the optical edge sensor measurement, which is local to the PM and insensitive to global motions of the optics, $c$ captures effects of all the optics as they affect pointing. The pointing measurement equation is:

$$
c_{i}=\frac{\partial c}{\partial x} x_{i}+c_{A t m}+c_{0}+\delta c_{i}
$$

Pointing measurements will be conducted at a lower rate than optical edge sensor measurements, at 0.001 to $0.01 \mathrm{~Hz}$.

\section{SIMULATION AND COVARIANCE ANALYSIS}

The performance of the 25-m CCAT can be bounded from the model equations outlined above, using simulation techniques and covariance analysis. Covariance analysis forms the basis of the CCAT Optical State Estimator discussed in the next Section.

In simulation, telescope performance is computed for a for a particular case, by initializing the state $x=x_{0}$ with random values consistent with the expected alignment and figure quality of the telescope, and then integrating the state forward in time using the transition equation (Eq. 2). At each time step, new state noise values $\xi$ are added, and values for $w, l$ and $c$ are computed. New controls (with sensing and actuation errors) can be fed back into the state via actuations $u$. The simulation is run until a steady-state performance is reached. The error in the final performance is a function of the particular errors and noise values that drive it. 
Monte Carlo analysis runs many simulations, each with the noise and errors generated using the same statistics, but with different particular realizations of these statistics, and then takes statistics on the results of all of the simulations, to understand the likely performance of a telescope with those particular component error statistics. By changing the component statistics, running more simulations and assessing those results, general conclusions can be drawn on performance as a function of the component error and noise performance statistics. Monte Carlo simulation and related simulation-based techniques are powerful tools, especially where statistics are not gaussian or the plant is nonlinear.
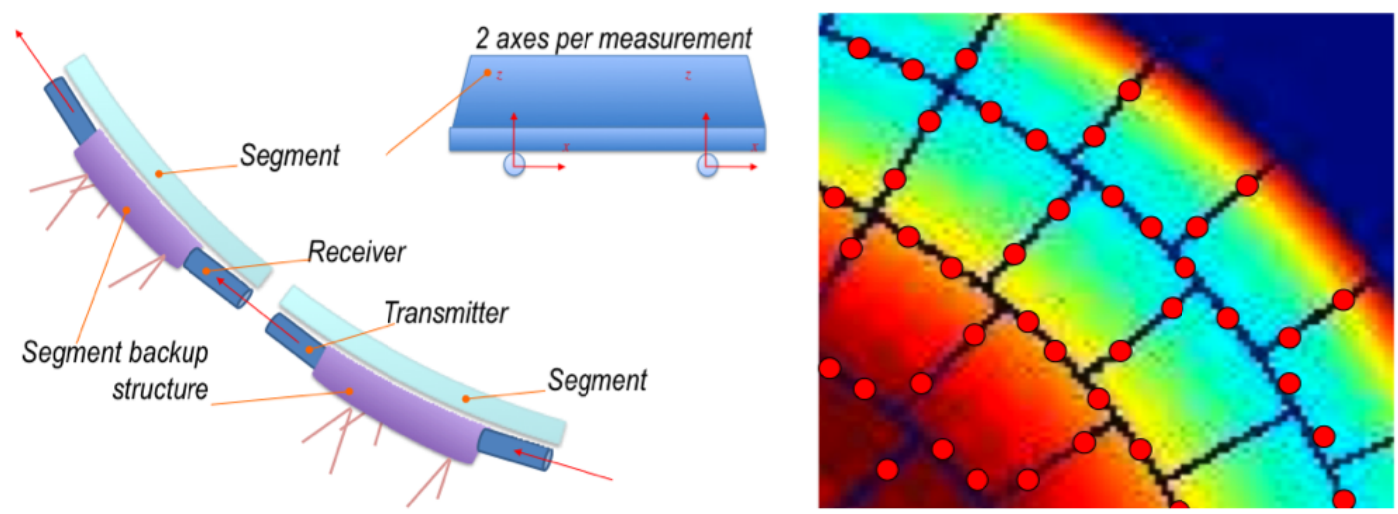

Figure 3. Optical edge sensor geometry and placement.

In covariance analysis, rather than conducting many simulations and then taking statistics on the results, the error statistics (in the form of covariance matrices) are propagated directly. Covariance analysis of a linear system provides the same result as Monte Carlo analysis, but with a single evaluation. Covariance analysis assumes that the errors in $x$ and $u$, the process noise $\xi$, and the measurement errors $\delta l$, are gaussian random variables, which is a good assumption for the small errors typical of telescopes. The errors are treated as zero-mean, by incorporating systematic effects into the underlying models. The process defined by these assumptions is a Markov process [8].

For a linear gaussian random vector $x$, the following general transformation holds [8]. If a vector $y=H x$, then y is also a linear gaussian random vector, with covariance $Y$, where

$$
Y=H X H^{T}
$$

In general we will denote the covariance of a random vector as the capital letter of that variable. Thus $\operatorname{cov}(x)=X, \operatorname{cov}(w)$ $=W, \operatorname{cov}(u)=U, \operatorname{cov}(\xi)=\Xi$, etc. Applying the rule of Eq. 8 to Eq. 5 , we get the state covariance transition equation:

$$
X_{i+1}=X_{i}+\frac{\partial x}{\partial u_{R}} U_{R i}\left[\frac{\partial x}{\partial u_{R}}\right]^{T}+\frac{\partial x}{\partial u_{S M}} U_{S M i}\left[\frac{\partial x}{\partial u_{S M}}\right]_{i}^{T}+\Xi_{i}
$$

This is a discrete Lyapunov equation. The covariance of the $i^{\text {th }}$ wavefront is:

$$
W_{i}=\frac{\partial w}{\partial x} x_{i}\left[\frac{\partial w}{\partial x}\right]^{T}+W_{A t m}
$$

The corresponding RMS WF error is

$$
W F E_{i}=\sqrt{\operatorname{trace}\left(\left(W_{i}\right) / n_{\text {ray }}\right)}
$$

Here $n_{\text {ray }}$ is the number of WF samples. Similar algebra is applied to develop expressions for the full closed-loop control WFE in the next Section.

Initial state errors $x_{0}$ will typically be generated from a combination of modes $T$, which produces a systematic, correlated but deterministic global shape change; and a random term that captures uncorrelated local effects. The initial covariance $X_{0}$ is therefore uncorrelated, diagonal, with the diagonal elements equal to the variance of the corresponding state as 
determined from component-level specifications. If $\varphi_{i}$ is the standard deviation or "1-sigma" error expected for the initial $x_{j}$ state vector element, then the corresponding entry in $X_{0}$ is:

$$
X_{0}(i, i)=1 / \sigma_{i}^{2}
$$

The state covariance matrix $X_{\mathrm{i}+1}$ is diagonal only at the initial condition: a single iteration of the control derived below cause the states to become correlated. The optical edge sensor measurement errors have a calibrateable static offset $l_{0}$ and uncorrelated measurement noise $\delta l$, with standard deviation $\sigma_{L}$, so that the covariance $L$ is:

$$
L_{0}=\left(1 / \sigma_{L}^{2}\right) I
$$

Here $\sigma_{L}$ is the (scalar) standard deviation of the error expected for optical edge sensor measurements, and $I$ is the identity matrix of dimension $n_{\text {ray }}$ by $n_{\text {ray. }}$. Similar expressions govern the other measurement covariances.

\section{OPTICAL STATE ESTIMATOR}

The optical edge sensor measurements $l$ (and $c$ and $w$ when available), can be used to estimate the optical state $x$ of the telescope at any particular time; and that estimate can be used to compute new controls $u_{R}$ and $u_{S M}$. This is the function of the Optical State Estimator (OSE): to take in all of the available data, and to process it to determine the underlying optical state $x$ of the telescope. The data that is continuously available includes the measurements $l$ and their covariance $L$; pointing measurements $c$ with covariance $C$; actuations $u_{R}$ and $u_{S M}$ and their error covariances $U_{R}$ and $U_{S M}$; and the covariance of the process noise, $\Xi$.

The OSE combines this data with a prediction of the state based on the previous state estimate to carry over information from earlier measurements. The formally optimal way to do this is by Kalman Filter techniques: weighted least-squares, where the estimate weights the measurements and the prior knowledge using the covariance matrices associated with each term to find the most likely state estimate [8], [9].

The Kalman Filter (KF) is a Maximum-Likelihood linear system estimator in recursive form, which allows it to produce estimates that are optimal at the time each new measurement is taken, while preserving the value of all previous measurements. The KF is a predictor-corrector estimator, where at each measurement we make a prediction of the measurement, based on the information we had at the last measurement, and compare that to the actual current measurement. The difference of the predicted and actual measurements, termed the innovation, is multiplied by a gain matrix and fed back to update the estimate. The gains are weighted by the covariances of the measurement and priorestimate errors, so as to achieve an optimal blending of prior and current information.

The prediction is made by taking the estimate computed at the preceding time step and simply projecting it forward in time using Eq. 2. We know the value of the control we commanded, so that goes into the prediction as well. The predicted measurement is then calculated by projecting the predicted state to the WF space using Eq. 3. Denoting the predicted state at time $i$ with an over-bar as, and the estimated state with a hat, as, the predicted state is:

$$
\bar{x}_{i+1}=\hat{x}_{i}+\frac{\partial x}{\partial u} u_{i}
$$

The predicted measurement is thus:

$$
\bar{l}_{i}=\frac{\partial l}{\partial x} \bar{x}_{i}
$$

The new state estimate is simply the prediction plus the innovation weighted by a gain matrix $K_{i}$ :

$$
\hat{x}_{i}=\bar{x}_{i}+K_{i}\left(l_{i}-\bar{l}_{i}\right)
$$

The gain balances the contribution of the innovation to the current estimate as the ratio of the error in the predicted state, as captured in the predicted-state covariance $P_{i}$, to the error in the measurement projected back to the state space via the transpose of the measurement matrix. We treat the actuator commands as known signals with a measurement error equal 
to the actuation noise projected to the WF space. The error in the measurement is the covariance matrix $R_{i}$, which includes the WF measurement error and the actuation error . The gain is:

$$
K_{i}=P_{i}\left(\frac{\partial l}{\partial x}\right)^{T} R_{i}^{-1}
$$

Note that the gain matrix $K_{l i}$ changes with every time step, which can impose a large computational burden on the OSE. In practice, however, $K$ will quickly approach a nearly static steady state, so that it is possible to precompute a set of $K_{i}$ matrices for the various different control conditions, and use them while those conditions obtain. The factors that cause $K_{i}$ to vary include variations in the noise covariances $L$ and $C$.

The measurement error covariance, which captures the uncertainty in both the actuation and the measurement, is:

$$
R_{i}=\frac{\partial l}{\partial x} \frac{\partial x}{\partial u} U_{i}\left(\frac{\partial l}{\partial x} \frac{\partial x}{\partial u}\right)^{T}+L_{i}
$$

The error in the estimate is captured by its covariance $P i$, which combines the measurement and predicted-state error covariances $R i$ and $M i$ in the state space, as:

$$
P_{i}=\left[\left(\frac{\partial l}{\partial x}\right)^{T} R_{i} \frac{\partial l}{\partial x}\right]^{-1}=\operatorname{cov}\left(\mathscr{x}_{i}\right)
$$

The covariance of the predicted state is computed based on the covariances of the error in the previous estimate, projected forward to the current time:

$$
M_{i+1}=P_{i}+\frac{\partial x}{\partial u} U_{i}\left(\frac{\partial x}{\partial u}\right)^{T}+\Xi_{i}
$$

Implementation of the Kalman update (Eq. 16), after substitutions, typically takes the form:

$$
\hat{x}_{i}=\left(I-K_{i} \frac{\partial l}{\partial x}\right) \hat{x}_{i-1}+\left(I-K_{i} \frac{\partial l}{\partial x}\right) \frac{\partial x}{\partial u} u_{i-1}+K_{i} l_{i}
$$

For gaussian normal random processes, the KF estimator of Eqs. 16 or 21 produces optimal estimates, in the sense that they make the best possible use of the available information, producing estimates with minimal error. In fact, the KF defines the Cramer-Rao bound for such processes [8]. In practical problems the KF assumptions are not always precisely met. The statistics of the processes may not be normal or stationary, the plant may not be truly linear. Many extensions to the KF have been developed to deal with specific situations of this type. However, for small deviations from the assumptions underlying the KF, the KF provides an excellent, though not precisely optimal, tool.

Equations 14-21 define the KF updates provided by the optical edge sensors, which occur at every cycle of the OSE. Other measurements, namely the pointing measurements $c$ and the WF measurements $w$ are incorporated in the same way, but at different times: the pointing measurements will be made every 10-1,000 cycles of the OSE; the WF measurements perhaps once a week. When such measurements are available, they are used to update the state $x$ in exactly the same way. For example, when a pointing measurement is available, the updated state estimate is:

$$
\hat{x}_{i}=\bar{x}_{i}+K_{c i}\left(c_{i}-\bar{c}_{i}\right)
$$

The gain and updated state estimate error covariance are:

$$
K_{c i}=P_{c i}\left(\frac{\partial c}{\partial x}\right)^{T} C_{c i}^{-1}
$$




$$
P_{c i}=\left[\left(\frac{\partial c}{\partial x}\right)^{T} R_{c i} \frac{\partial c}{\partial x}\right]^{-1}=\operatorname{cov}\left(\frac{Q}{X_{i}}\right)
$$

Similar results obtain for WF updates through measurements of $w$.

To illustrate the effect of the gain matrix $\mathrm{K}$ in balancing prior estimates versus current measurements, it is useful to consider 2 extreme cases. The first case occurs when the error in the prior estimate is very small, and very much smaller than the measurement noise, so that $P_{i}$ approaches 0 . Then $K_{i}$ approaches 0 , and the estimate is simply the prediction:

$$
\hat{x}_{i} \rightarrow \bar{x}_{i}
$$

The other extreme case occurs when the combined measurement noise is very much less than the error in the prior estimate. The gain (Eq. 17) can be rewritten using the inversion lemma as:

$$
K_{i}=P_{i}\left(\frac{\partial l}{\partial x}\right)^{T}\left[R_{i}+\frac{\partial l}{\partial x} P_{i}\left(\frac{\partial l}{\partial x}\right)^{T}\right]^{-1}
$$

Now when $R_{i}$ approaches 0 ,

$$
K_{i} \rightarrow\left[\left(\frac{\partial l}{\partial x}\right)^{T} \frac{\partial l}{\partial x}\right]^{-1}\left(\frac{\partial l}{\partial x}\right)^{T}
$$

which is the familiar pseudo-inverse solution of the measurement equation (Eq. 6).

The best performance for any real case, where prior knowledge error and measurement noise are in more nearly the same, is obtained when the covariances $X_{0}, \Xi, L$, and $C$ accurately represent the real errore, as they determine the balance between measurement and prior knowledge for the OSE. If the measurement noise covariance reflects an assumed small measurement error, but the actual measurement error is large, for instance, the $K_{i}$ matrix will couple too much of the measurement error into the state estimate, and performance of the OSE will be degraded - see example in Figure 5.

\section{WAVEFRONT CONTROLLER}

The best way to control the WFE is to directly measure $w$ and use those measurements to compute new control values. The cost function for this form of control is:

$$
\min _{u} J=w^{T} w+c_{u} u^{T} u
$$

There are 2 terms here, balanced by a scalar coefficient $c_{u}$. The first term is the square of the WF; the second is the square of the control effort between the $i^{\text {th }}$ and the $i-1^{\text {th }}$ iteration. The coefficient can be tuned to balance the control effort against the WFE, a useful knob to turn in modulating the control response. The control law that satisfies this cost function is:

$$
u_{i}=-\left[c_{u} I+\left(\frac{\partial w}{\partial x} \frac{\partial x}{\partial u}\right)^{T} \frac{\partial w}{\partial x} \frac{\partial x}{\partial u}\right]^{-1}\left(\frac{\partial w}{\partial x} \frac{\partial x}{\partial u}\right)^{T} w_{i}
$$

Continuous direct measurement of $w$ is not provided under the current CCAT baseline. Instead, the state estimate produced by the OSE can be used to estimate $w$ :

$$
\bar{w}_{i}=\frac{\partial w}{\partial x} \hat{x}_{i}
$$


The resulting "Wavefront Compensation" control law is:

$$
u_{i}=-G x_{i}
$$

Here the gain matrix $G$ is:

$$
G=\left[c_{u} I+\left(\frac{\partial w}{\partial x} \frac{\partial x}{\partial u}\right)^{T} \frac{\partial w}{\partial x} \frac{\partial x}{\partial u}\right]^{-1}\left(\frac{\partial w}{\partial x} \frac{\partial x}{\partial u}\right)^{T} \frac{\partial w}{\partial x}
$$

Using the estimated state as the basis for CCAT control allows the controller to use actuation in the controlled DOFs to mitigate the optical effects of errors that are incurred in non-actuated DOFs. An example case is the lateral translation of a segment. Such a motion occurs when the base structure expands, and the consequence is a small but significant WFE, as the segment moves off of the ideal surface. This error occurs in a direction mechanically - but not optically orthogonal to the 3DOFs along which the actuators act. By moving the segment in piston and tilt, the optical effects of this displacement can be largely corrected.

\section{EXAMPLES}

A single-step simulation example is illustrated in Figure 4. This example illustrates estimation performance for a large initial segment state error randomly generated, with 50 um or 50 urad standard deviation per axis, including the uncontrolled axes such as segment lateral translation. Optical edge sensor noise in this case was $1 \mathrm{um}(0.1 \mathrm{um}$ sensing noise is expected). The results show good estimation and good WF control performance $(\lambda / 100$ at the minimum $\lambda)$.
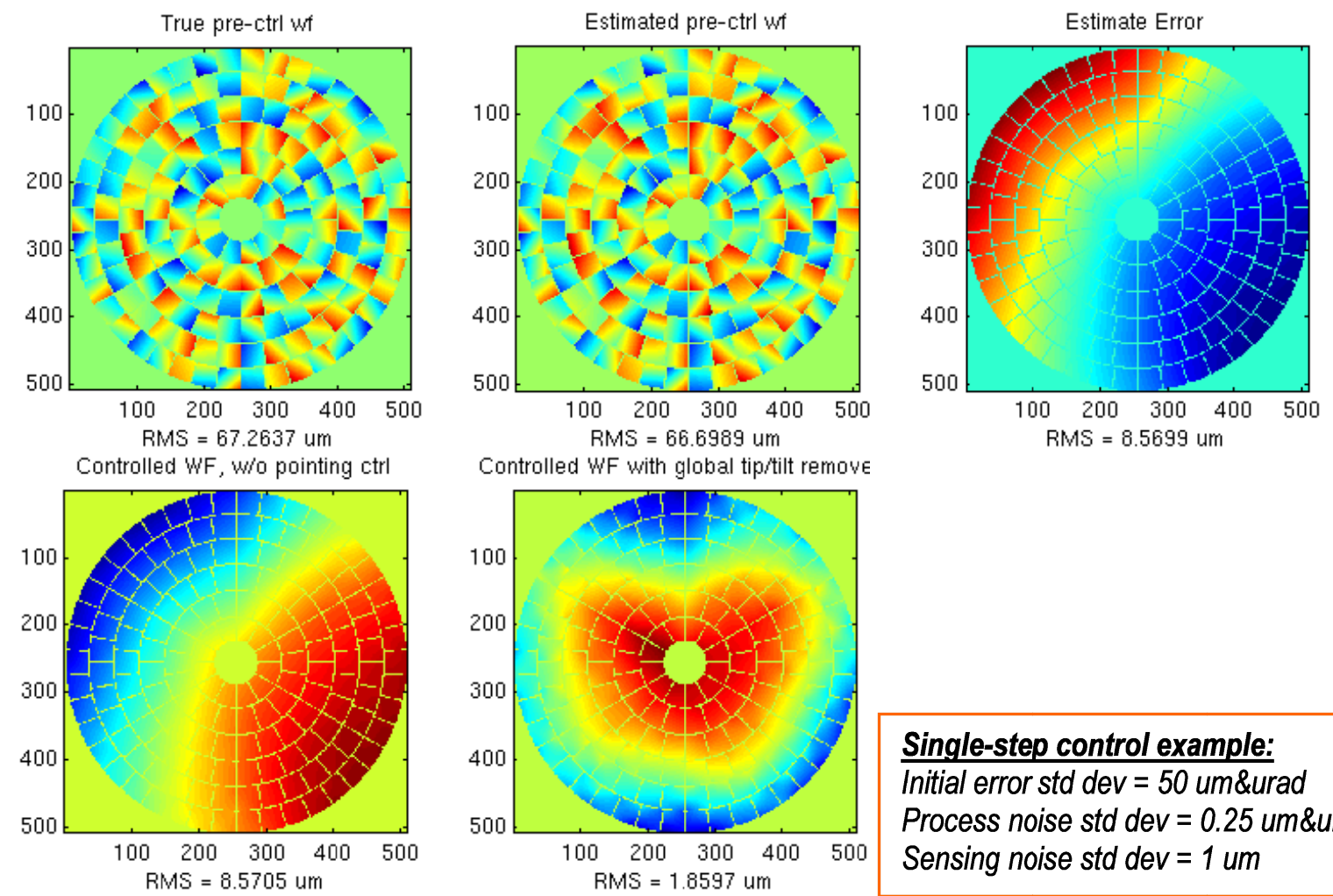

\section{Single-step control example: Initial error std dev $=50$ um\&urad Process noise std dev $=0.25$ um\&urad Sensing noise std dev $=1$ um}

Figure 4. Single-step control example, showing actual WF, the WF estimated from optical edge sensor measurements, and the estimation error; plus the controlled WF with and without global tilts.

Figure 5 shows Monte Carlo results, where many cases were run with the same initial error statistics (50 um or 50 urad standard deviation per axis), but with different sensing noise and different assumed sensing noise, $R_{0}$. Each solid curve 
reperesents the WFE after control using a gain matrix K generated assuming a single value of the sensing noise standard deviation (" $R$ "). Note that each curve provides the best WFE performance when $R$ is in balance with the actual sensing error. The locus of all best cases - with $R$ consistent with actual sensing noise - is approximated by the dashed curve. As shown, in the range of expected sensing noise performance, the WFE is $2.5 \mathrm{um}$, at the noise floor determined by the initial errors.

The final example illustrates the use of the control damping parameter $c_{u}$, which is used to weight the control effort against the WFE in the cost function $J$ (Eq. 28). Larger values of $c_{u}$ slow the response of the controller, making it less sensitive to noise in the state estimate.

\section{WFE vs. Sensing Noise}

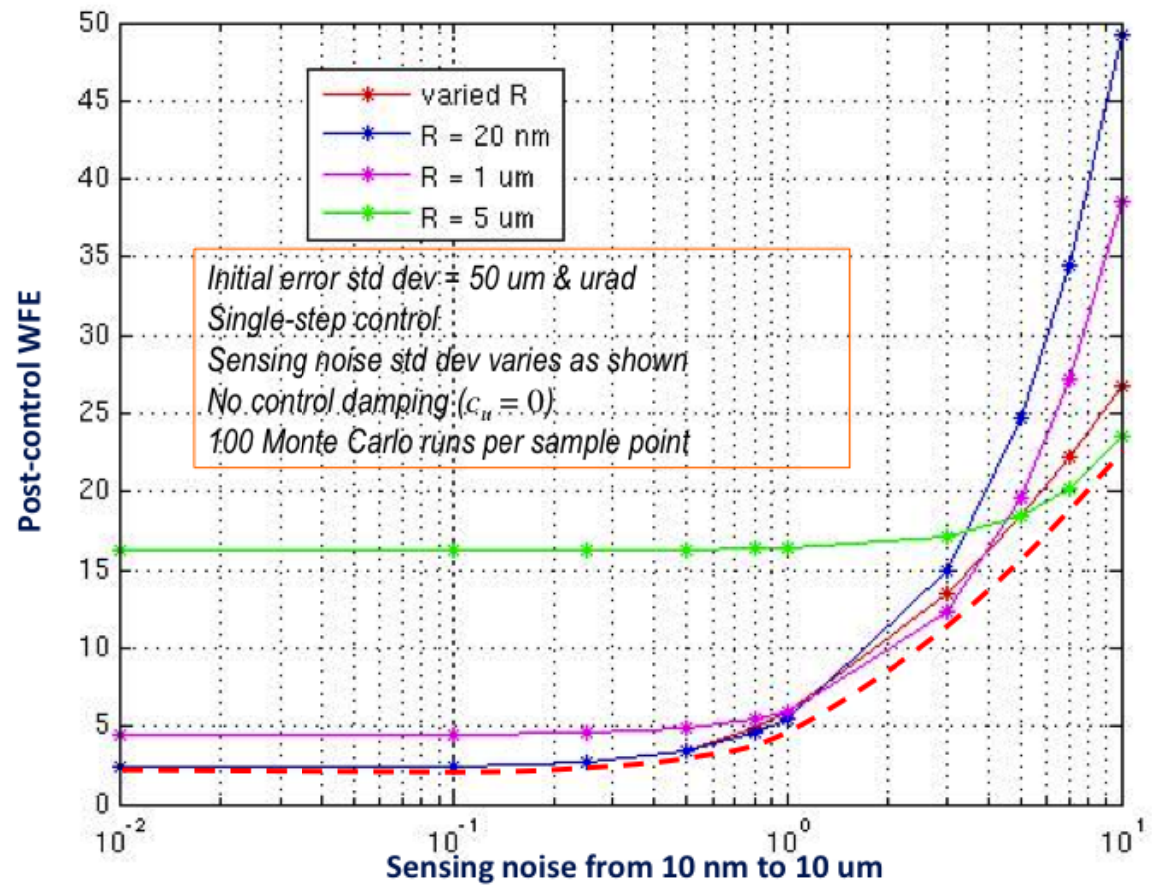

Figure 5. Post-control WFE vs. sensing noise standard deviation: the different curves each have different assumed sensing noise standard deviation $R$; the best performance is obtained when the assumed and actual sensing noise is approximately equal (red dashed line). At the expected level of $0.1 \mathrm{um}$, WFE $<3 \mathrm{um}$.
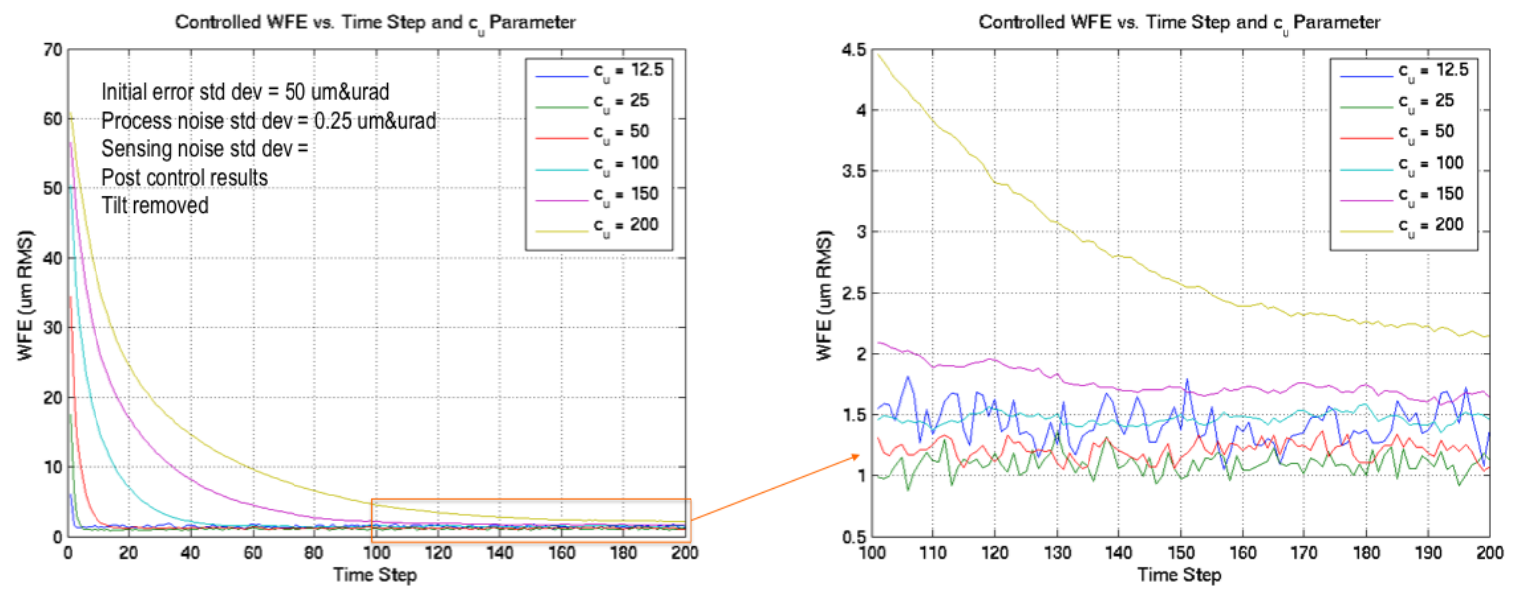

Figure 6. WFE for 200 time steps, showing recovery from initial state errors of 50 um or $50 \mathrm{urad}(1 \sigma)$ for different values of the control damping parameter $c_{u}$. 


\section{CONCLUSION}

The CCAT "Wavefront Compensation" maintenance controller provides continuous wavefront maintenance control through full state feedback. It incorporates an Optical State Estimator to estimate the optical state and wavefront error based on optical edge sensor measurements, and then uses this estimate in a controller explicitly designed to minimize wavefront error. This design permits effective control, not only of errors in the directly-controlled degrees of freedom, but also of the optical effects of errors in uncontrolled degrees of freedom. Control performance can be tuned by selection of noise parameters in the state estimator gain computation, and by use of control damping terms in the control gain computation. Preliminary results indicate that control performance will be good, meeting CCAT requirements, for a representative set of errors. It remains to fully explore the range of CCAT operating conditions. Further work will refine and update the models of CCAT to add dynamics, to incorporate design changes, to provide a thorough performance assessment, and to support more detailed design trade studies.

This research was carried out at the Jet Propulsion Laboratory, California Institute of Technology, under a contract with the National Aeronautics and Space Administration.

\section{REFERENCES}

[1] Cornell Caltech Atacama Telescope (CCAT) Feasibility and Concept Design Study, Final Report (2006).

[2] E. Serabyn, T. Phillips and C. Masson, "Surface Figure Measurements of Radio Telescopes with a Shearing Interferometer," Applied Physics, Vol. 30, No. 10 (1991).

[3] J.Z. Lou, A. Kissil, D. Redding, C.M. Bradford, S.Padin and D. Woody, "Modeling a Large Submillimeter-Wave Observatory," SPIE (2010).

[4] MACOS Manual, JPL Document (1999).

[5] D. Redding and W. Breckenridge, "Linearized Ray-Trace Analysis," Selected SPIE Papers on CD ROM, Volume 2, Lens Design, D. O'Shea, ed. (1998).

[6] A. Abramovici, "Electro-Optic Measurement of CCAT Segment-Segment and Primary-Secondary Relative Motions," JPL Internal Document (2009).

[7] D. MacDonald, D. Woody, C. Bradford, R. Chamberlin, M. Dragovan, S. Radford, T. Sebring, J. Zmuidzinias, and P. Goldsmith, "Cornell Caltech Atacama Telescope Primary Mirror Surface Sensing and Controllability," SPIE 7012, (2008).

[8] A. Bryson and Y. Ho, Applied Optimal Control, Hemisphere Publishing (1975).

[9] D. Redding, B. Breckenridge, Ken Lau, G. Sevaston, M. Levine and S. Shaklan, "Segmented Mirror Figure Control for a Space-Based Far-IR Astronomical Telescope,” SPIE 1489 (1991). 